\title{
Variation Morphologique Adaptative Des Populations De Sarotherodon Melanotheron Des Secteurs IV Et V De La Lagune Ebrié (Côte d'Ivoire) Face Au Stress Chimique
}

\author{
Bopo Zadi Sylvain Olivier
}

Etudiant-Doctorant, Laboratoire de Génétique, UFR Biosciences, Université Félix Houphouët-Boigny, Abidjan, Côte d'Ivoire

\section{Sokouri Didier Paulin}

Maitre de Conférences, Laboratoire de Génétique, UFR Biosciences, Université Félix Houphouët-Boigny, Abidjan, Côte d'Ivoire

\section{Nobah Céline Sidonie Koco}

Maitre de Conférences Science et Technique, Section Sciences de la Vie et de la Terre, Ecole Normale Supérieure (ENS), Abidjan, Côte d'Ivoire

\section{Atsé Boua Célestin}

Directeur de Recherche, Centre de Recherches Océanologiques (CRO), Abidjan, Côte d'Ivoire

\begin{abstract}
The impact of chemical pollution on organism's characteristics was assessed through morphological features in IV and V sectors of Ebrié Lagoon using Sarotherodon melanotheron (Rüppel, 1852) as bioindicator. Sampling was carried out during a whole year in three sites (Layo, N'djem and Ahua) characterized by contrasted environments(respectively moderate, medium and high levels of pollution). The morphological studies were based on morphometric biomarkers such as fluctuating asymmetry (FA) and average body shape. Fluctuating asymmetry was evaluated among individuals within sites using ANOVA Procrustes. Average body shape was compared among sampling sites using Principal Components Analysis (PCA), Canonical Variates Analysis (CVA) and MANOVA. Fluctuating asymmetry did not vary between sampling sites. However, the average body shape was significantly different between specimens from Ahua and both those from Layo and N'djem. There was no significant difference between fish from Layo and those from N'djem.Allometric effects and the abiotic parameters influenced, to some extent, the body shape of individuals. A morphological differentiation according to the geographical distance was also observed. The study revealed
\end{abstract}


a morphological adaptation of the populations of $S$. melanotheron in the study area.

Keywords: Sarotherodon melanotheron, chemical stress, fluctuating asymmetry, body shape

\section{Résumé}

L'impact de la pollution chimique sur les caractéristiques biologiques des organismes des secteurs IV et V de la lagune Ebrié a ètè évalué à travers des traits morphologiques chez Sarotherodon melanotheron (Rüppel, 1852) comme bioindicateur. L'échantillonnage s'est déroulé durant une année entière dans trois sites (Layo, N'djem et Ahua) caractérisés par des environnements contrastés (niveaux de pollution modéré, moyen et très élevé respectivement). Les études morphologiques ont été menées à partir des biomarqueurs morphométriques tels que l'asymétrie fluctuante et la conformation corporelle moyenne. L'asymétrie fluctuante a été évaluée à travers une Procrustes ANOVA tandis que les conformations corporelles moyennes ont été comparées entre individus par sites d'échantillonnage à 1'aide d'une Analyse en Composantes Principales (ACP), d'une Analyse Canonique Discriminante (CVA) et d'une Analyse Multivariée de Variance (MANOVA). Il ressort que les valeurs d'asymétrie fluctuante ne varient pas entre sites d'echantillonnage. Cependant, les conformations corporelles moyennes restent significativement différentes entre individus de Ahua et ceux de Layo et de N'djem. Aucune différence significative entre les poissons de Layo et ceux de N'Djem. En outre, les effets allométriques et les paramètres abiotiques ont, dans une certaine mesure, une influence sur la conformation corporelle moyenne des individus. Une différenciation morphologique en fonction de la distance géographique a été également observée. L'étude a conclu l'existence d'une adaptation morphologique des populations de $S$. melanotherondans dans la zone étude.

Mots-clés: Sarotherodon melanotheron, stress chimique, asymétrie fluctuante, conformation corporelle

\section{Introduction}

Les écosystèmes aquatiques se trouvent être le réceptacle final de la majorité des contaminants chimiques issus des activités anthropiques (Depledge \& Galloway, 2005). Parmi ces écosystèmes, les lagunes et les estuaires subissent les plus fortes variations des facteurs abiotiques et donc d'intenses pressions écologiques et socioéconomiques constantes (Klein \& Nicholls, 1999). En Côte d'Ivoire, la lagune Ebrié ne fait pas exception à cette règle. Depuis octobre 1999, cette lagune est soumise de façon récurrente, dans 
ses secteurs IV et $\mathrm{V}$, à des mortalités massives de poissons du fait de la pollution chimique des eaux (Boni, 2016). La pollution chimique pouvant jouer un rôle important dans les pressions de sélection naturelle contemporaines (Postma et al., 1995), il est possible de s'attendre à des changements microévolutifs au niveau des populations naturelles. Ainsi, en complément des analyses chimiques et écologiques déjà proposées à ce sujet (Boni, 2017), l'utilisation de biomarqueurs morphométriques tels que l'asymétrie fluctuante et la forme corporelle peut aider à mesurer les réponses phénotypiques d'un stress environnemental au niveau individuel et à l'échelle de la population (Leamy \& Klingenberg, 2005). L'asymétrie fluctuante est une petite déviation aléatoire à partir d'une symétrie bilatérale parfaite qui résulte d'un stress génétique et / ou environnemental durant le développement (Van Valen, 1962). C'est est un marqueur extérieur de l'instabilité du développement, qui est définie comme une suite de processus qui tendent à perturber la précision du développement. Aujourd'hui, ces biomarqueurs parviennent à avoir un large spectre d'application en s'appuyant sur la morphométrie géométrique, qui fournit des perspectives interessantes pour l'étude de la biodiversité (Alibert et al., 2001).

Le tilapia Sarotherodon melanotheron a été choisi comme espèce modèle pour illustrer les conséquences potentielles de ce stress chimique. Ce taxon est beaucoup représenté dans les secteurs IV et V de la lagune Ebrié (Boni, 2016), zone de couverture de cette étude En outre, il est sensible aux variations des conditions climatiques et environnementales, pouvant potentiellement jouer le rôle d'espèce sentinelle (Van de Oost et al., 2003; McIntyre et al., 2010). Ainsi, l'objectif de cette étude est d'évaluer l'influence du stress chimique, qui sévit dans les secteurs IV et V de la lagune Ebrié, sur les caractéristiques morphologiques des populations de $S$. melanotheron par l'utilisation de bioindicateurs tels que l'asymétrie fluctuante et la variation de la forme corporelle.

\section{Matériel et Méthode \\ Milieu d'étude}

La lagune Ebrié (Figure 1) est le plus grand écosystème côtier de 1'Afrique de l'ouest (Guiral \& Ferhi, 1989). Elle est située au Sud de la Côte $\mathrm{d}^{\prime}$ Ivoire $\left(5^{\circ} 13^{\prime} 15^{\prime \prime} \mathrm{N}\right.$ et $4^{\circ} 424^{\prime} 0^{\prime \prime}$ ' $\left.\mathrm{W}\right)$ avec une profondeur moyenne de $4,8 \mathrm{~m}$ et s'étend sur $130 \mathrm{~km}$ le long de la façade littorale (Varlet, 1978). Cette zone est caractérisée par trois saisons, la saison sèche (de janvier à avril), la saison des pluies (de mai à août) et la saison des crues (de septembre à décembre) (Durand \& Guiral, 1994). Sur la base de critères hydrobiologiques, cette lagune a été divisée en six secteurs (Durand \& Guiral, 1994). La zone d'étude se situe dans les secteurs IV et V, dans les départements de Dabou et de Jacqueville. Trois sites d'échantillonnage ont été retenus : Layo, N'djem et 
Ahua. La caractérisation de ces sites en termes de niveau de stress environnemental est basée sur les conclusions d'un rapport d'enquête préliminaire du Ministère des ressources animales et halieutiques (MIRAH) relatif à la mortalité des organismes aquatiques enregistrée en Mai 2013 à Dabou et à Jacqueville. Les conclusions de ce rapport d'enquête ont été confirmées plus tard par la détermination du niveau de pollution organique et de certains indices caractéristiques du peuplement ichtyologiques (indices de comparaison d'Abondance-Biomasse; indices de Clarke) selon les travaux de Boni (2016). Ainsi, le site de Layo (4²1'16,72"N et $\left.5^{\circ} 18^{\prime} 14,55^{\prime \prime O}\right)$ a été considéré comme modérément pollué, le site de $\mathrm{N}^{\prime}$ djem $\left(4^{\circ} 13^{\prime} 26,52^{\prime \prime} \mathrm{N}\right.$ et $\left.5^{\circ} 16^{\prime} 57,74^{\prime \prime} \mathrm{O}\right)$ comme moyennement pollué et le site de Ahua $\left(4^{\circ} 27^{\prime} 41,00^{\prime \prime} \mathrm{N}\right.$ et $\left.5^{\circ} 13^{\prime} 52,84^{\prime \prime O}\right)$ comme très pollué.

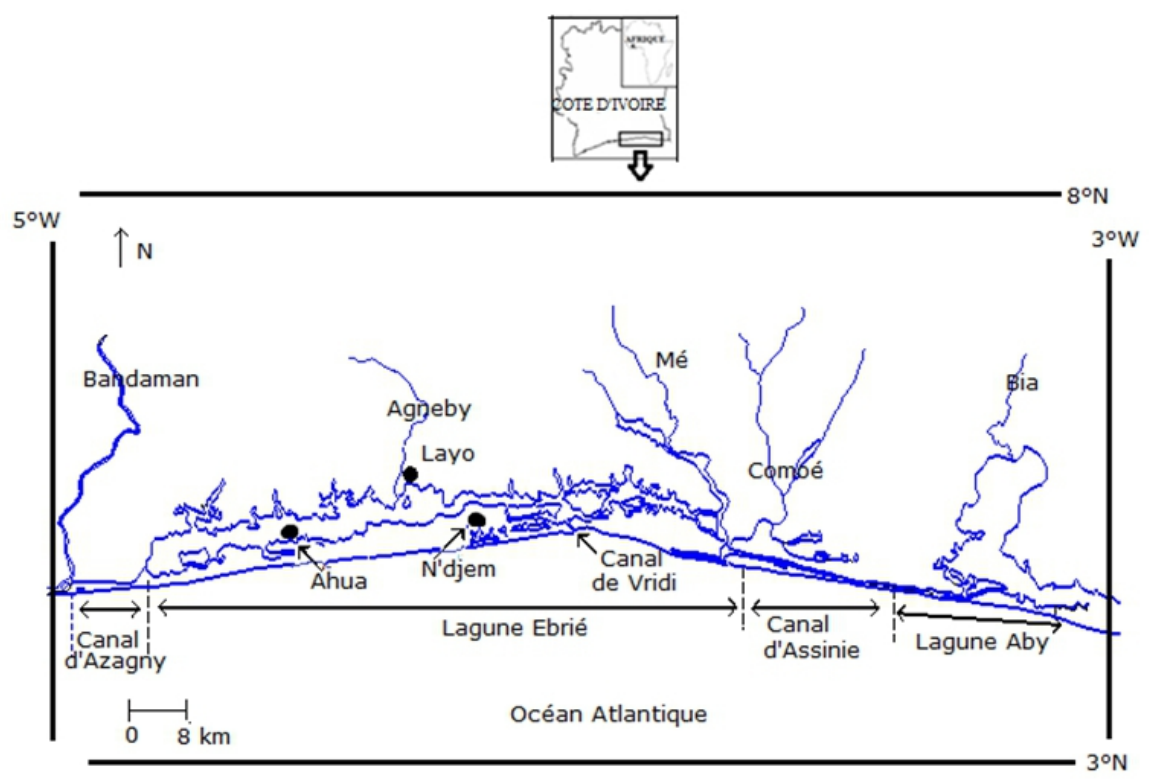

Figure 1: Complexe lagunaire Ebrié et localisation des sites d'échantillonnage $(\bullet)$

\section{Echantillonnage ichtyologique}

L'échantillonnage a été effectué de février 2014 à janvier 2015, durant trois jours consécutifs, au cours des campagnes de sorties mensuelles, effectuées par une équipe pluridisciplinaire sur les effets des changements environnementaux de la lagune Ebrié de Dabou à Jacqueville. Les pêches se sont déroulées dans les secteurs IV (Layo) et V (N'Djem et Ahua) de la lagune Ebrié. Les individus de Sarotherodon melanotheron ont été capturés par une équipe de trois pêcheurs par site d'échantillonnage. Plusieurs engins de pêche ont été utilisés : les filets maillants de vide de mailles $40 \mathrm{~mm}$, les éperviers de mailles variant entre 10 et $60 \mathrm{~mm}$ et les sennes de plage de mailles comprises entre 12 et $20 \mathrm{~mm}$. Ensuite, les spécimens de poissons par sites d'échantillonnage ont été conditionnés pour la prise des mesures 
morphométriques. Au total, 80 poissons ont été considerés par site d'échantillonnage avec une égalité de mâles et femelles.

\section{Mesure des paramètres physico-chimique de l'eau}

Les caractéristiques physico-chimiques de l'eau ont été mesurées parallèlement à la collecte des échantillons de poissons. Les différentes mesures ont été effectuées in situ, une fois par mois, entre $7 \mathrm{~h}$ et $9 \mathrm{~h}$ du matin. Pour ce faire, un multiparamètre de type YSI 6920 a été utilisé pour mesurer la température, la salinité, l'oxygène dissous et le $\mathrm{pH}$ selon les instructions du fabricant. La valeur finale de chaque paramètre était la moyenne de trois mesures successives.

\section{Morphométrie géométrique}

La morphométrie est l'étude de la variation de la forme, des traits anatomiques des organismes et des co-variations qui en découlent, en relation avec d'autres variables d'intérêt comme le régime alimentaire, la taille, etc. (Bookstein, 1991 ; Dryden \& Mardia, 1998). La forme simplifiée au sens géométrique d'un objet biologique au temps t est composée de deux éléments ; la taille (l'échelle de l'objet) et la conformation (l'information spatiale hors échelle intrinsèque à l'objet). La morphométrie géométrique étudie séparement la conformation et la taille après l'application des procédures de superposition. La variable de la taille qui en découle est la taille centroïde et les variables de conformation sont les partial warps scores ou leur composantes principales (les relative warps scores). En outre, pour illustrer la variation de conformation entre des populations, la distance Procrustes peut être aussi utilisée. Cette distance est estimée par la racine carrée de la somme des carrés des distances entre les éléments des deux conformation (Zelditch et al., 2004). L'étude des points repères homologues reste l'approche la plus utilisée en morphométrie géométrique pour l'étude des formes. Les points repères sont définis comme étant des locus anatomiques discrets qui peuvent être identifiés chez tous les spécimens de l'étude (Zelditch et al., 2004). Chaque point repère est associé à des coordonnées cartésiennes en deux ou trois dimensions. L'analyse porte sur les coordonnées relatives de ces points qui permettent de capturer la géométrie de l'objet à l'étude.

Les méthodes de morphométrie géométrique ont été trouvées pertinentes dans plusieurs champs d'étude y compris la stabilité de développement (Klingenberg \& McIntyre, 1998). L’asymétrie fluctuante, signe extérieur de l'instabilité de développement, n'est pas la seule forme d'asymétrie au niveau de la forme des organismes. Il y a également l'asymétrie directionnelle (AD) et l'antisymétrie (AS). Ces deux types d'asymétrie illustrent l'état d'une structure bilatérale où l'asymétrie est la norme. Dans un cas d'AD, le côté privilégié de chaque individu est généralement le même dans 
une population. Par contre il est aléatoire dans le cas de l'AS (Van Valen, 1962).

\section{Positionnement des points repères}

La prise des mesures morphométriques a debuté par le positionnement des points repères sur les poissons. Concernant l'étude de la forme corporelle moyenne, 19 points repères ont été considérés (Figure 2a). De préférence, chaque poisson a été placé horizontalement sur une surface plane. Par convention, seule la face latérale gauche du poisson est prise en compte de sorte que la tête est dirigée à gauche et la queue à droite. Concernant l'asymétrie fluctuante, 11 points repères, placés respectivement sur chacune des faces latérales gauche et droite du poisson ont été considérés (Figure 2b). Ces points repères sont symétriques par rapport au plan qui sépare en deux parties égales les faces du poisson. Les mesures morphométriques ont été effectuées à partir des photographies prises avec un appareil numérique de marque Samsung, model $\mathrm{N}^{\circ} \mathrm{WB}$ 200F. Lors de la prise de vue, celui-ci est positionné au-dessus de chaque préparation. La distance verticale entre l'objectif et la préparation est restée constante à $35 \mathrm{~cm}$ à toutes les prises d'image en conservant la même luminosité et le même agrandissement.
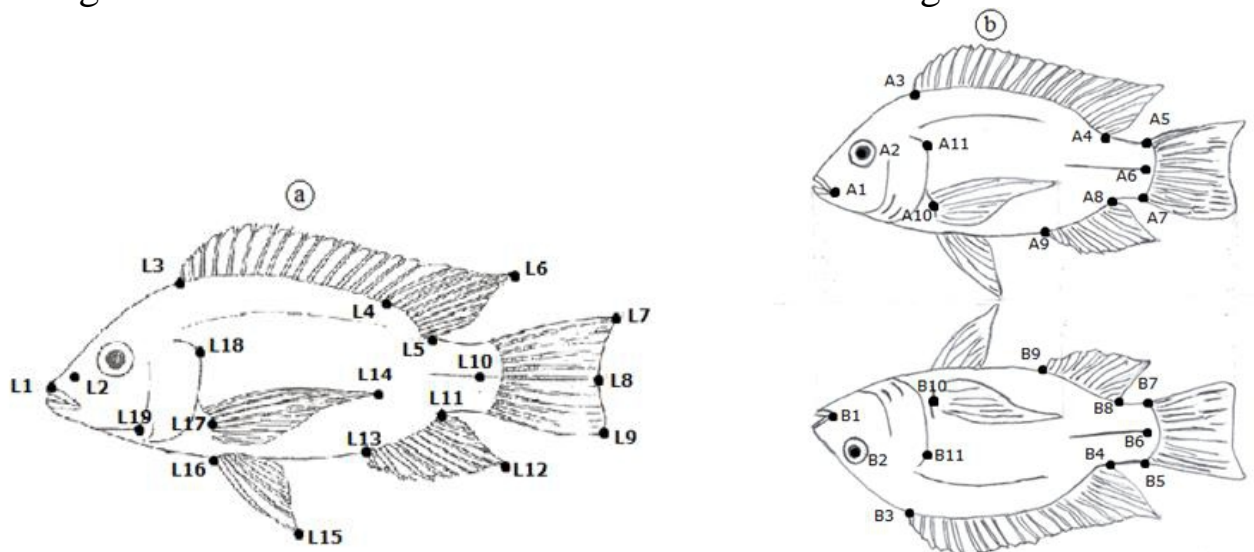

Figure 2: Emplacement des points repères sur les poissons

L1: Sommet de la lèvre supérieure; L2: Ouverture du nez; L3, A3, B3: Insertion antérieure de la nageoire dorsale; L4: Base postérieure du dernier rayon dur de la nageoire dorsale; L5, A4, B4: Insertion postérieure de la nageoire dorsale; L6: Sommet des rayons mous de la nageoire dorsale; L7: Extrémité antérieure de la nageoire caudale; L8: Limite postérieure de la nageoire caudale; L9: Extrémité postérieure de la nageoire caudale; L10, A6, B6: Limite postérieure de la ligne latérale; L11, A8, B8: Insertion postérieure de la nageoire anale; L12: Extrémité supérieure de la nageoire anale; L13, A9, B9: Insertion antérieure de la nageoire anale; L14: Extrémité supérieure de la nageoire pectorale; L15: Extrémité inférieure de la nageoire pelvienne; 
L16: Insertion antérieure de la nageoire pelvienne; L17, A10, B10: Extrémité inférieure de la nageoire pectorale; L18, A11, B11: Point le plus élevé de la courbure de l'opercule; L19, A12, B12: Point le plus bas de la courbure de la mâchoire inférieure; A1, B1: Limite de l'ouverture de la bouche; A2, B2: Centre de l'œil; A5, B5: Limite dorsale de la nageoire dorsale; A7, B7: Limite ventrale de la nageoire dorsale

\section{Numérisation des points repères et superposition des configurations}

Pour l'étude de la forme corporelle moyenne, les points repères ont été numérisés et leurs coordonnées déterminées grâce au logiciel tps DIG (Rohlf, 2003). Les différentes configurations des poissons ont été ensuite superposées selon la méthode Procrustes, qui utilise la procédure des moindres carrés. Cette étape a permis un alignement de ces configurations à travers une rotation, une translation et d'une mise à l'échelle des coordonnées des points repères. La superposition permet ainsi de placer les configurations des points repères dans un même référentiel tout en supprimant les effets de position et de taille des spécimens afin de les comparer aisément. La superposition Procrustes a été réalisée grâce au programme CoorGEN6 de la série IMP (Sheet, 2004)

Concernant l'AF, les configurations de points repères provenant d'une face latérale du poisson ont d'abord subi une réflexion de sorte à les superposer aux configurations respectives de l'autre face latérale. Cet ensemble a été ensuite soumis à un ajustement Procrustes, qui permet un alignement des spécimens dans un même système de coordonnées, dans lequel les variations interindividuelles et intra-individuelles peuvent être examinées (Klingenberg \& McIntyre, 1998).

\section{Analyse statistique des données \\ Etude de la forme corporelle moyenne}

La comparaison des conformations corporelles moyennes entre groupes de spécimens provenant respectivement de Layo, Ahua et N'djem a été faite successivement par une Analyse en Composantes Principales (ACP), par une Analyse Canonique discriminante (CVA) et par une analyse multivariée de variances (MANOVA). Ces analyses ont été effectuées à l'aide des logiciels PCAGen6, CVAGen6 de la série IMP (Sheet, 2004) et PAST (Hammer et al., 2005), respectivement. Lorsque la MANOVA a montré des différences significatives entre les groupes, des comparaisons multiples deux à deux des conformations ont été réalisées sur les distances généralisées de Mahalanobis. Les valeurs significatives ont été obtenues après correction de Bonferroni au seuil de 5\%. Les effets allométriques qui correspondent à la variation de la conformation due à la taille ont été déterminés à l'interieur de chaque groupe de spécimens grâce au logiciel Regress6k de la série IMP 
(Sheet, 2004). Cette analyse a consisté en une régression multiple des variables de conformation (partial warp scores) sur la matrice des tailles centroïdes..

L'influence des paramètres physico-chimiques sur la conformation corporelle des individus de Sarotherodon melanotheron a été testée par une analyse en Partial Least Square (PLS) en utilisant le logiciel PLSMaker6 de la série IMP (Sheet, 2004). L'analyse PLS est une technique de corrélation multivariée qui décrit la covariation entre deux ensembles ou deux blocs de variables. Cette analyse peut être utilisée pour explorer des patrons de covariation entre la conformation et d'autres variables telles que les paramètres environnementaux, les traitements expérimentaux ou d'autres conformations de diverses structures (Rolhf \& Corti, 2000).

Un test de Mantel a été utilisé pour établir la corrélation entre les matrices de distances morphologiques (distance Procrustes) et les matrices de distances géographiques entre les sites d'échantillonnage. Le test de Mantel a été réalisé grâce au logiciel PAST (Hammer et al., 2005)

\section{Etude de l'AF}

Les sommes de carrés des déviations provenant de la configuration moyenne, obtenue après l'ajustement Procrustes à travers les coordonnées de tous les points repères ont été établies à travers une Procrustes ANOVA (Klingenberg \& McIntyre, 1998).

La Procrustes ANOVA permet d'accéder au niveau de significativité de potentielles sources de variation. Le côté ou face latérale du poisson est pris comme variable fixe et l'individu (spécimen de poisson), pris comme variable aléatoire. Les valeurs des sommes des carrés, sommées et ajustées, des sources de variation "individus", "côté" et "interaction individu x côté" correspondent respectivement, aux valeurs de la variabilité inter-individuelle, d'asymétrie directionnelle et d'asymétrie fluctuante. L'ajustement Procrustes et la Procrustes ANOVA ont été réalisés au moyen du logiciel SAGE version 1.04 (Marquez, 2007). Toutefois, l'estimation de l'AF peut être biaisée par la présence des deux autres formes d'asymétrie (AD et AS), par l'allométrie et par l'erreur de mesure (Palmer, 1994). Il faut donc en quantifier les valeurs. 


\section{Résultats}

\section{Comparaison des conformations corporelles moyennes par ACP}

Les spécimens sont projetés dans le plan CP1 - CP2 qui rend compte de $61,78 \%$ de la variabilité totale (Figure 3). Deux regroupements de spécimens se distinguent. Un premier est composé des spécimens de Sarotherodon melanotheron provenant de Layo et de N'djem. Ces individus surtout situés dans la partie négative de l'axe CP1. Le second regroupement est uniquement constitué des individus venant de Ahua. Ces spécimens sont à l'opposé de ceux du premier groupe sur l'axe CP1. Cependant, ces deux regroupements ont la même position sur l'axe CP2.

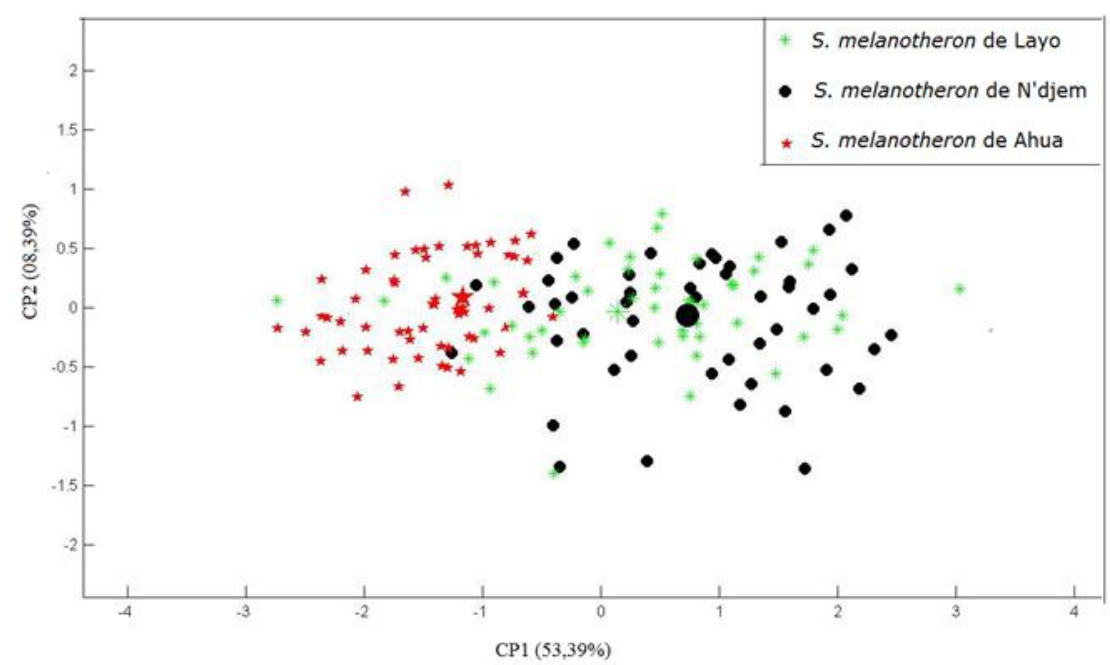

Figure 3 : Projection des spécimens de Sarotherodon melanotheron provenant de Layo,

N'djem et Ahua sur les deux premiers axes de l'analyse en Composantes Principales

\section{Comparaison des conformations corporelles moyennes par CVA}

Les individus de $S$. melanotheron ont été projetés dans le plan for mé par les axes CV1 et CV2 expliquant $73 \%$ de la variabilité totale. Trois regroupements d'individus se distinguent (Figure 4). Un premier groupe, formé par les spécimens de Layo, occupent quasiment le plan (-CV1;+CV2). Le second groupe est constitué des individus venant de $\mathrm{N}^{\prime}$ djem. Ils sont repérés en grande partie dans le plan (-CV1; -CV2). Ces deux premiers groupes possèdent quelques individus en commun sur l'axe (-CV1). Le dernier groupe est constitué des individus de Ahua. Ils sont situés dans le plan $(+\mathrm{CV} 1$; $+\mathrm{CV} 2)$. 


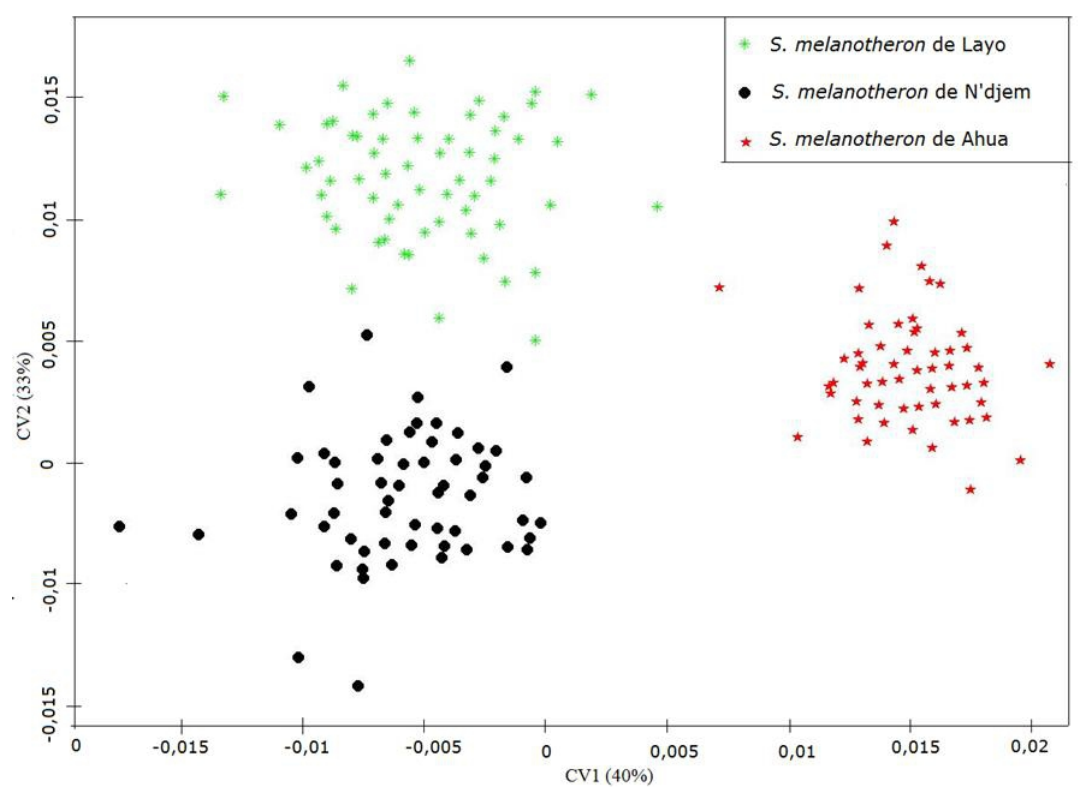

Figure 4: Projection des spécimens de Sarotherodon melanotheron de Layo, N'djem et Ahua sur les deux premiers axes de l'analyse canonique discriminante

\section{Comparaison des formes corporelles moyennes par MANOVA}

La MANOVA (Wilk's lambda $=0,000132, \mathrm{~F}_{340,520}=3,98, p=0,003$ présente une différence significative des conformations moyennes. Les comparaisons deux à deux montrent que les spécimens de Ahua sont significativement différents de ceux de Layo $(\mathrm{F}=12,75, p=0,009$ et de $\mathrm{N}$ 'djem $(\mathrm{F}=11,80, p=0,008)$. Cependant, il n'y a pas de différence significative entre les spécimens de Layo et ceux de N'Djem ( $\mathrm{F}=2,15, p=0,321)$.

\section{Détermination de l'allométrie}

L'allométrie est significative $(p<0,05)$ dans la variation de la conformation corporelle des individus de $S$. melanotheron provenant des différents sites d'échantillonnage. Cependant la part exprimée par la taille sur la variation de la conformation reste faible dans tous les échantillons (Tableau 1).

\begin{tabular}{cccc}
\hline Sites d'échantillonnage & $\mathrm{F}$ & $\begin{array}{c}\text { Pourcentage } \\
\text { expliquée }\end{array}$ & $\mathrm{p}$ \\
\hline Ahua & 2,40 & 7,07 & 0,04 \\
Layo & 2,09 & 6,16 & 0,03 \\
N'djem & 2,13 & 6,26 & 0,04 \\
\hline
\end{tabular}

Tableau 1: Tests multivariés des régressions linéaires des variables de conformation en fonction de la taille centroïde de Sarotheron melanotheron des sites d'échantillonnage 


\section{Corrélation distance morphologique-distance géographique}

Le test de Mantel indique que les distances morphologiques entre les sites de Layo, N'djem et Ahua sont significativement corrélées aux distances géographiques respectivement $(p=0,007)$. Le coefficient de corrélation est positif et très élevé $(r=0,90)$.

Corrélation morphologie-variables abiotiques

La première paire des axes singuliers concentre la plus grande partie de la covariation entre les variables abiotiques et la morphologie des individus. La corrélation entre ces deux blocs estpositive et significative $(p<0,05)$, mais elle reste moyenne dans l'ensemble.

\begin{tabular}{|c|c|c|c|c|c|c|c|c|c|c|c|c|}
\cline { 2 - 14 } \multicolumn{1}{c|}{} & \multicolumn{3}{c|}{ Température } & \multicolumn{4}{c|}{$\mathrm{pH}$} & \multicolumn{3}{c|}{ Oxygène dissous } & \multicolumn{3}{c|}{ Salinité } \\
\hline Sites & $r$ & SW1 & $p$ & $r$ & SW1 & $p$ & $r$ & SW1 & $p$ & $r$ & SW1 & $p$ \\
\hline Layo & 0,43 & 99 & 0,02 & 0,56 & 99 & 0,03 & 0,54 & 85,54 & 0,02 & 0,48 & 99 & 0,01 \\
\hline N`djem & 0,45 & 99 & 0,01 & 0,45 & 99 & 0,01 & 0,52 & 96,73 & 0,03 & 0,47 & 99 & 0,01 \\
\hline Ahua & 0,37 & 99 & 0,01 & 0,43 & 99 & 0,01 & 0,52 & 86,40 & 0,02 & 0,41 & 99 & 0,02 \\
\hline
\end{tabular}

Le coefficient de corrélation (r), pourcentage de variance expliquée et la significativité de la corrélation entre la première paire de Singular Warps $(S W)$ ont été représentés.

Tableau 2: Résultat de l'analyse en Partial Least Square (PLS) entre sites d'échantillonnage (Layo, Ahua, N'djem) et paramètres abiotiques

\section{Détermination de l'asymétrie fluctuante}

Le manque d'agrégation des nuages de points représentant les vecteurs de la différence des faces gauches et droites des poissons montre l'absence de l'antisymétrie dans les conformations respectives des poissons pour chaque site d'échantillonnage (Figure 5). Les différentes régressions entre les vecteurs d'asymétrie et les tailles centroïdes respectives montre l'absence d'allométrie dans les asymétries fluctuantes des différentes conformations (Layo : $\mathrm{F}=$ 0,225; N'djem : $\mathrm{F}=0,270$; Ahua :, $\mathrm{F}=0,158 ; P<0,05$ pour tous les sites).

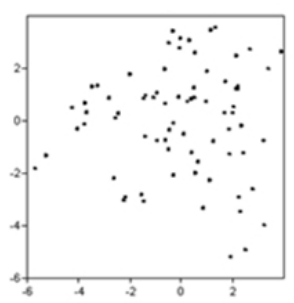

S. melanotheron de Ahua
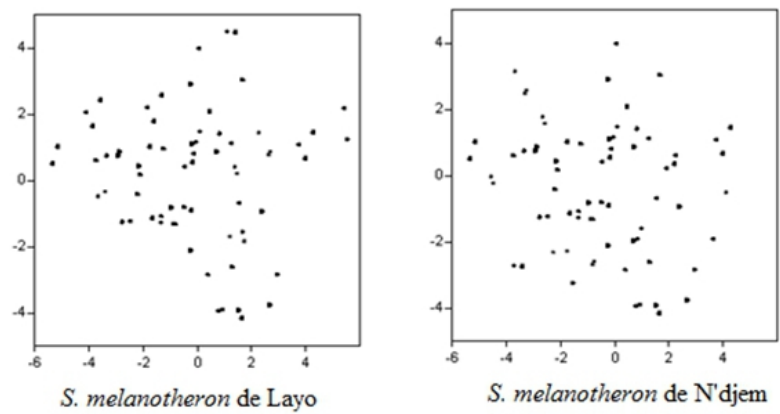

Figure 5 : Nuages de points représentant les vecteurs de la différence des faces gauches et droites des spécimens de poissons de Ahua, Layo et N`djem 
Les résultats de la Procrustes ANOVA de conformation montrent que les variances liées aux effets " individu", "côté" et interaction "individu $\mathrm{x}$ côté" sont significatives $(p<0,05)$. Ceci montre que la variance interindividuelle, l'asymétrie directionnelle et l'asymétrie fluctuante sont significatives. Toutefois, la valeur de l'asymétrie fluctuante $(0,0001)$ est similaire dans tous les sites d'échantillonnage (Tableau 3).

\begin{tabular}{|c|c|c|c|c|c|}
\hline Effets & $\mathrm{SC}$ & ddl & $\mathrm{CM}$ & $\mathrm{F}$ & $P$ \\
\hline \multicolumn{6}{|c|}{ Sarotherodon melanotheron de Ahua } \\
\hline Individu & 0,0441 & 280 & 0,0002 & $1,8745^{*}$ & 0,01 \\
\hline côté & 0,0563 & 20 & 0,002 & $33,5313 *$ & 0,02 \\
\hline $\begin{array}{l}\text { Individu x } \\
\text { côté }\end{array}$ & 0,0235 & 280 & 0,0001 & $16,8798 *$ & 0,01 \\
\hline $\begin{array}{l}\text { Erreur de } \\
\text { mesure }\end{array}$ & 0,013 & 600 & 0 & -- & -- \\
\hline \multicolumn{6}{|c|}{ Sarotherodon melanotheron de Layo } \\
\hline Individu & 0,1685 & 500 & 0,0003 & $2,8954 *$ & 0,01 \\
\hline côté & 0,0352 & 20 & 0,0018 & $14,6423 *$ & 0,01 \\
\hline $\begin{array}{l}\text { Individu x } \\
\text { côté }\end{array}$ & 0,0601 & 500 & 0,0001 & $8,9728 *$ & 0,01 \\
\hline $\begin{array}{l}\text { Erreur de } \\
\text { mesure }\end{array}$ & 0,0139 & 1040 & 0 & -- & -- \\
\hline \multicolumn{6}{|c|}{ Sarotherodon melanotheron de N'djem } \\
\hline Individu & 0,1622 & 580 & 0,0003 & $2,2109^{*}$ & 0,01 \\
\hline côté & 0,0272 & 20 & 0,0014 & $10,7566 *$ & 0,01 \\
\hline $\begin{array}{l}\text { Individu } \mathrm{x} \\
\text { côté }\end{array}$ & 0,0734 & 580 & 0,0001 & $9,5653 *$ & 0,01 \\
\hline $\begin{array}{l}\text { Erreur de } \\
\text { mesure }\end{array}$ & 0,0159 & 1200 & 0 & -- & -- \\
\hline
\end{tabular}

SC : Somme des carrés $;$ ddl $:$ dégré de liberté; $\mathrm{CM}:$ carré moyen; $*: \mathrm{P}<0,05$, le dégré de significativité a été testé par permutation avec 1000 répétitions.

Tableau 3: Résultats de la Procrustes ANOVA de conformation

\section{Discussion}

Sarotherodon melanotheron a été utilisée comme espèce modèle pour évaluer la modification des caractéristiques biologiques des organismes aquatiques des secteurs IV et V de la lagune Ebrié face au stress chimique. Cette étude s'est basée sur l'un des principes selon lequel, l'étude de la variation de la forme moyenne entre groupes d'organismes de la même espèce, soumis à différents niveaux de stress peut illustrer l'impact du stress sur les organismes aquatiques (Klingenberg et al., 2001 ; Hoffmann et al., 2002). La forme moyenne de $S$. melanotheron a été étudiée dans trois sites d'échantillonnage caractérisés par des niveaux de stress différents (Layo: faiblement pollué, N'djem: moyennement pollué et Ahua: très pollué). Il ressort que la forme de $S$. melanotheron varie entre les sites de Layo/N'djem et Ahua. Cette variation morphologique est fonction de la distance géographique selon les résultats du test de Mantel. La forme des individus de 
S. melanotheron est, dans une certaine mesure, également influencée par les paramètres abiotiques étudiés (température, $\mathrm{pH}$, salinité et oxygène dissous) et par la taille des individus selon les analyses respectives en partial least square et l'étude de l'allométrie. Ces différents résultats pourraient suggérer l'idée d'une variation génétique en cours entre les populations de $S$. melanotheron. Etant donné la durée et le caractère fortement letal du stress chimique qui sévit dans la zone d'étude, le développement des organismes a dû être très influencé. Il est possible que les mécanismes de corrections des traits morphologiques engagés par les processus d'homéostasie du développement aient été inefficaces. Ceci pourrait se traduire par la production de phénotypes différents au niveau morphologique. Emlen \& Nijhout (2000) soutiennent qu'une perturbation au cours du développement peut se traduire par la production de phénotypes différents, surtout que les substances polluantes dans le milieu aquatique sont considérées comme des éléments perturbateurs majeurs pouvant agir sur le processus de développement des organismes (Klingenberg et al., 2001; Hoffmann et al., 2002). Cette production de différents phénotypes au niveau individuel ou familial suivant l'environnement commence généralement par la plasticité phénotypique, qui peut être adaptative ou non selon les organismes. Cependant, lorsque le stress environnemental est de grande ampleur et reste persistant sur une longue période, la plasticité phénotypique reste limitée, du fait du coût énergétique lié au mécanisme et à la régulation nécessaire pour assurer un tel niveau de flexibilité (DeWitt et al., 1998). Il peut en résulter une forte mortalité des individus plastiques et dans une moindre mesure, la baisse globale de la reproduction et de la croissance. Il peut également y avoir une adaptation par sélection de génotypes tolérants de sorte que les individus les possédant développent des stratégies de survie. Il est possible que cette situation soit celle des populations de $S$. melanotheron dans la zone étude où des mortalités massives d'organismes aquatiques sont observées depuis plusieurs années.

L'asymétrie fluctuante (AF) a été également utilisée pour comparer le niveau d'instabilité du développement chez des populations exposées à un stress environnemental tel que la pollution chimique (Hoffmann \& Woods, 2003; Møller \& Swaddle, 1997). Pour toutes ces études, l'AF est supposée plus élevée chez les populations les plus stressées. Cependant, dans cette étude, les asymétries fluctuantes ne varient pas entre sites d'échantillonnage, ce qui ne semble pas logique étant donné les niveaux de stress divergents qui ont été observés. Les individus échantillonnés sur le site Ahua devraient avoir la valeur d'asymétrie fluctuante la plus élevée sur la base de la forte pollution des eaux constatées dans ce lieu. Ce résultat laisse suggérer soit une adaptation locale des individus de $S$. Melanotheron, soit l'homogénéisation des divergences entre populations par des flux géniques. En effet, dans le cadre d'études portant sur des populations naturelles collectées dans différents 
environnements stressants, Hoffmann et Woods (2003) ont montré que l'absence de relation entre l'asymétrie fluctuante et le stress peut être la conséquence d'une adaptation. Ces auteurs soutiennent qu'au sein d'une population qui s'est adaptée à un environnement stressant, les génotypes "adaptés" devraient avoir un développement plus stable que les génotypes "non adaptés", avec pour conséquence une AF moyenne respectivement plus faible. D'autre part, les sites d'échantillonnage étant géographiquement proches et non séparés par des obstacles majeurs, des échanges éventuels d'adultes, d'embryons ou de gamètes ont dû avoir lieu, limitant les différences entre eux. Cependant, les différences dans les conformations corporelles moyennes entre sites d'échantillonnage semblent plaidées en faveur d'une adaptation locale. Des analyses de différenciation génétique neutre $\left(\mathrm{F}_{\mathrm{ST}}\right)$ et de différenciation génétique quantitative $\left(\mathrm{Q}_{\mathrm{ST}}\right)$ pourraient permettre de révéler le mécanisme évolutif majeur responsable des patrons observés.

\section{Conclusion}

La pollution chimique de longue durée qui sévit dans les secteurs IV et $\mathrm{V}$ de la lagune Ebrié pourrait être à la base de l'adaptation locale des populations de Sarotherodon melanotheron, constatée dans cette étude. Des changements génétiques en cours pourraient être à l'origine de cette différenciation morphologique. Des études de génétiques moléculaires devraient être faîtes à ce sujet afin de préciser ce résultat.

\section{Remerciement}

Les auteurs tiennent à remercier le FIRCA (Fonds Interprofessionnel pour la Recherche et le Conseil Agricole) pour l'appui financier apporté pour la réalisation de ce travail. En effet, cette étude a été réalisée dans le cadre du projet relatif à l'effet des changements environnementaux sur la vulnérabilité des ressources halieutiques de la lagune Ebrié, de Jacqueville à Dabou. Les auteurs remercient également, les chercheurs et toutes autres personnes ayant participé à la collecte des données.

\section{References:}

1. Alibert, P., Moureau, B., Dommergues J.L., \& David, B. (2001). Differenciationat a microgeographical scale within two species of ground beetle, Carabus auronitens and C. nemoralis (Coleoptera, Carabidae): a geometrical morphometric approach. The Norwegian Academy of Science and Letters. Zoologica Scripta 30 (4): 299-311.

2. Anger, K. (2006). Contributions of larval biology to crustacean research: a review. Invertebrate Reproduction and Development 49:175-205.

3. Boni, L. (2016). Physical and chemical parameters and nutrients in the 
Ebrié Lagoon (Côte d'Ivoire, west Africa): Impact on fish mortality. International Journal of Research In Earth \& Environmental Sciences. $4(3): 1-16$.

4. Boni, L. (2017). Diversité et structure des peuplements des poissons dans deux secteurs lagunaires: cas des secteurs IV et $\mathrm{V}$ de la lagune Ebrié (Côte d'Ivoire). Thèse Unique de Doctorat, Université Houphouet Boigny. Abidjan, Côte d'Ivoire, 197 p.

5. Bookstein, F.L. (1991). Morphometric tools for landmark data: geometric and biology. Cambridge University Press, 435 p.

6. Depledge, M. H., \& Galloway, T. S. (2005). Healthy animals, healthy ecosystems. Frontiers in Ecology and the Environment. 3 (5): 254-258.

7. DeWitt, T. J., Sih, A., \& Wilson, D. S. (1998). Costs and limits of phenotypic plasticity. Trends in Ecology \& Evolution 13:77-81.

8. Dryden, I.L., \& Mardia, K.V. (1998). Statistical shape analysis. New York: John Wiley and Sons. 200 p.

9. Durand, J. R., \& Guiral, D.(1994). Hydroclimat et hydrochimie. In : Durand J. R., Dufour P.,Guiral D. \& Zabi S. G. F. (Eds.). Environnement et Ressources Aquatiques de Côted'Ivoire : Les Milieux Lagunaires (Tome II). Paris, France: ORSTOM. pp 59-90.

10. Emlen, D. J., \& Nijhout, H. F. (2000). The development and evolution of exaggerated morphologies in insects. Annual Review of Entomology 45, 661-708.

11. Guiral, D. \& Ferhi A. (1989). Caractérisation ionique et isotopique d'un système hydrologique tropical: la lagune Ebrié (Côte d'Ivoire). Oceanologica Acta, 12(1): 47-55.

12. Hammer, D.A., \& Ryan, P.D. (2005). PAST: Paleontology software package for education and data analysis. Paleontologia Electronica 4: 1.

13. Hoffmann, A. A., Collins, E., \& Woods, R. (2002). Wing shape and wing size changes as indicators of environmental stress in Helicoverpa punctigera (Lepidoptera: noctuidae) moths: comparing shifts in means, variances, and asymmetries. Environmental Entomology 31, 965-971.

14. Hoffmann, A. A., \& Woods, R. E. (2003). Associating environmental stress with developmental stability: problems and patterns, p. 387-401. In M. Polak (ed.), Developmental Instability: Causes and Consequences. Oxford University Press, Oxford.

15. Klein, R.J.T., \& Nicholls, RJ. (1999). Assessment of coastal vulnerability to climate change. Ambio 28, 182-187.

16. Klingenberg, C. P., \& McIntyre, G. S. (1998). Geometric morphometrics of developmental instability: analyzing patterns of fluctuating asymmetry with Procrustes methods. Evolution 52: 13631375 . 
17. Klingenberg, C. P., Badyaev, A. V., Sowry, S. M,. \& Beckwith, N. J. (2001). Inferring Developemental Modularity from Morphological Integration: Analysis of Individual Variation and Asymmetry in Bumblebee Wings. The American Naturalist 157, 11-23.

18. Leamy, L.J., \& Klingenberg, C.P (2005). The genetic and evolution of fluctuating asymmetry. Annual Review of Ecology, Evolution and Systematics, 1-21.

19. Marquez, E. (2007). SAGE: Symmetry and Asymmetry in geometric Data. Version 1.04.

20. McIntyre, K.M., Setzkorn, C., Baylis M., Waret-Szkuta, A., Caminade, C., Morse, A.P., \& Morand, S. (2010). Impact of climate change on human and animal health. Vetenary Resources 167: 586-586.

21. Møller, A. P., \& Swaddle, J. P. (1997). Asymmetry, developmental stability, and evolution. R.M May and P.H Harvey Ed. Oxford University Press. 291 p.

22. Postma, J. F., \& Davids, C. (1995). Tolerance induction and life cycle changes in cadmium-exposed Chironomus riparius (Diptera) during consecutive generations. Ecotoxicology \& Environmental Safety 30:195-202.

23. Rohlf, F. J. (2003). tpsDIG, version 1.38. Department of Ecology and Evolution, State University of New York at Stony Brook.

24. Rohlf, F. J., \& Corti, M. (2000). The use of two-block partial leastsquares to study covariation in shape. Systematic Biology 49:740-753.

25. Sheet, H.D. (2004). Morphometric software: IMP-Integrated Morphometric Software. Buffalo NY: Department of Physics, Canisius College.

26. Van der Oost., Beyer, R.J., \& Vermeulen, N.P. (2003). Fish bioaccumulation and biomarkers in environmental risk assessment: a review. Environmental Toxicology Pharmacology. 13, 57-149.

27. Van Valen, L. (1962). A study of fluctuating asymmetry. Evolution 16:125-142.

28. Varlet, F. (1978). Le régime de la lagune Ebrié (Côte d'Ivoire). Traits physiques essentiels. Travaux et documentations O.R. S.T.O.M. n83. $231 \mathrm{p}$.

29. Zelditch, M.L., Swiderski D.L., Sheets, H.D. \& Fink, W.L. (2004). Geometric morphometrics for biologists: A primer. Elsevier Academic Press: San Diego. 